\title{
MEAN CONVERGENCE AND COMPACT SUBSETS OF $L_{1}$
}

\author{
BENJAMIN HALPERN
}

ABSTRACr. It is shown that in the usual criterion for $w$ compactness of a set $K$ in $L_{1}(\mu)$ the explicit assumption that $K$ is bounded follows from the other assumptions which are usually made if $\mu$ is nonatomic.

We establish a result connecting the concepts of equicontinuity from above at $\phi$ and uniform absolute continuity for a collection of measures. We draw from this result sharper forms of known theorems on mean convergence and compact subsets of $L_{1}$.

In Paul Halmos' book Measure theory, there appears the following theorem (Theorem C, p. 108):

THEOREM. A sequence $\left\{f_{n}\right\}$ of integrable functions converges in the mean to the integrable function $f$ if and only if $\left\{f_{n}\right\}$ converges in measure to $f$ and the indefinite integrals of $\left\{f_{n}\right\}, n=1,2, \cdots$, are uniformly absolutely continuous and equicontinuous from above at $\phi$. (In the "if" direction the function $f$ is assumed measurable and it is part of the conclusion that $f$ is integrable.)

We will prove the following sharpening of this result in the "if" direction.

THEOREM 2. If a sequence $\left\{f_{n}\right\}$ of integrable functions converges in measure to a measurable function $f$ and the indefinite integrals of $\left\{f_{n}\right\}$, $n=1,2, \cdots$, are equicontinuous from above at $\phi$, then $f$ is integrable and $\left\{f_{n}\right\}$ converges in the mean to $f$.

Dunford and Schwartz prove in their book Linear operators. Part I the following theorem (Theorem 9, p. 292):

Theorem. $A$ subset $K$ of $L_{1}(S, \Sigma, \mu)$ is weakly sequentially compact if and only if it is bounded and the indefinite integrals of the $f$ in $K$ are equicontinuous from above at $\phi$.

This can be sharpened in the "if" direction in the special case where $\mu$ is atom free.

THEOREM 3. If $\mu$ is atom free then a sufficient condition for a subset

Received by the editors January 27, 1970.

AMS 1969 subject classifications. Primary 2820.

Key words and phrases. Mean convergence, $L_{1}$, equicontinuity, absolute continuity. 
$K \subset L_{1}(S, \Sigma, \mu)$ to be weakly sequentially compact is that the indefinite integrals of the $f$ in $K$ are equicontinuous from above at $\phi$.

Theorems 2 and 3 follow from the quoted theorems and the fact that the omitted hypothesis in each case can be shown to be a consequence of the remaining hypotheses. In fact this is the mode in which we will establish these results.

Proofs. Let $X$ be a set and $S$ a $\sigma$-ring of subsets of $X$. We recall that a collection $C$ of finite real or complex valued measures on $S$ are said to be equicontinuous from above at $\phi$, provided each decreasing sequence $\left\{E_{n}\right\}$ in $S$ with void intersection the $\operatorname{limit}_{n} \lim _{n} v\left(E_{n}\right)=0$ is uniform for $v \in C$. (Dunford and Schwartz in [1] refer to this notion as "the countable additivity of the measures being uniform with respect to $v \in C^{\prime \prime}$.)

Our first theorem is the central result of this paper from which the other results follow.

THEOREM 1. Let $X$ be a set, $S$ a $\sigma$-ring subsets of $X$ and $\mu$ a nonnegative real valued measure defined on $S$. If $C$ is a collection of finite real valued signed measures defined on $S$ whose members are individually absolutely continuous with respect to $\mu$ and equicontinuous from above at $\phi$ then the members of $C$ are uniformly absolutely continuous with respect to $\mu$.

Proof. We will prove this by contradiction. We assume that the members of $C$ are not uniformly absolutely continuous with respect to $\mu$. Under this hypothesis we can find a $\Delta>0$ and construct a sequence of sets $\left\{E_{n}\right\}$ and a corresponding sequence of measures $\left\{V_{n}\right\} \subset C$ such that

$$
\left|V_{n}\left(E_{n}\right)\right|>\Delta, \quad\left|V_{n}\left(\bigcup_{j=n+1}^{\infty} E_{j}-E_{n}\right)\right| \leqq \frac{1}{2} \Delta
$$

and

$$
\lim _{n \rightarrow \infty} \mu\left(\bigcup_{j=n}^{\infty} E_{j}\right)=0
$$

Then we will form the decreasing sequence $F_{n} \downarrow \phi$ by setting

$$
F_{n}=\bigcup_{j=n}^{\infty} E_{j}-\bigcap_{j=1}^{\infty} \bigcup_{l=j}^{\infty} E_{l} .
$$

The sequence $F_{n}$ will contradict the equicontinuity from above at $\phi$.

Now we proceed with the actual proof. Since each $V \in C$ is absolutely continuous with respect to $\mu$, for every $\epsilon>0$ and $V \in C$, there 
exists a $\delta_{V}(\epsilon)$ such that $E \in S$ and $\mu(E) \leqq \delta_{V}(\epsilon)$ implies $|V(E)| \leqq \epsilon$. Now assume that the members of $C$ are not uniformly absolutely continuous with respect to $\mu$. Then there is a $\Delta>0$ such that for each $d>0$ there exists a $V^{d} \in C$ and $E^{d} \in S$ such that

$$
\mu\left(E^{d}\right)<d \text { and }\left|V^{d}\left(E^{d}\right)\right|>\Delta .
$$

Now define $d_{n}, V_{n}$ and $E_{n}$ recursively by

$$
\begin{gathered}
d_{1}=\frac{1}{2}, \quad V_{1}=V^{d_{1}}, \quad E_{1}=E^{d_{1}}, \\
d_{n+1}=\min \left(d_{n} / 2, \delta_{V_{n}}(\Delta / 2) / 2\right), \\
V_{n+1}=V^{d_{n+1}}, \quad E_{n+1}=E^{d_{n+1}} .
\end{gathered}
$$

Next set $F_{n}=\bigcup_{j=n}^{\infty} E_{j}-\bigcap_{j=1}^{\infty} \bigcup_{l=j}^{\infty} E_{l}$. We claim that the $F_{n}$ contradict the equicontinuity from above at $\phi$ of the members of $C$. Clearly by (1)

$$
\left|V_{n}\left(E_{n}\right)\right|=\left|V^{d_{n}}\left(E^{d_{n}}\right)\right|>\Delta \text { for all } n .
$$

Also observe

$$
d_{j} \leqq\left(\frac{1}{2}\right)^{j-n} d_{n} \quad \text { for } j \geqq n \geqq 1
$$

and thus

$$
d_{j} \leqq\left(\frac{1}{2}\right)^{j-n-1} d_{n+1} \leqq\left(\frac{1}{2}\right)^{j-n} \delta_{V_{n}}(\Delta / 2) \quad \text { for } j>n \geqq 1 .
$$

Hence

and

$$
\sum_{j=n+1}^{\infty} d_{j} \leqq \delta_{V_{n}}\left(\frac{\Delta}{2}\right)
$$

$$
\sum_{j=n+1}^{\infty} d_{j} \leqq d_{n} \leqq\left(\frac{1}{2}\right)^{n-1} d_{1}=\left(\frac{1}{2}\right)^{n} \text { for } n \geqq 1 .
$$

Therefore, using (1) we have

$$
\begin{aligned}
\mu\left(\bigcup_{j=n+1}^{\infty} E_{j}\right) \leqq & \sum_{j=n+1}^{\infty} \mu\left(E_{j}\right)=\sum_{j=n+1}^{\infty} \mu\left(E^{d_{j}}\right) \\
& \leqq \sum_{j=n+1} d_{j} \leqq \min \left(\delta_{V_{n}}(\Delta / 2),\left(\frac{1}{2}\right)^{n}\right) .
\end{aligned}
$$

It follows that

$$
\mu\left(\bigcup_{j=n+1}^{\infty} E_{j}-E_{n}\right) \leqq \mu\left(\bigcup_{j=n+1}^{\infty} E_{j}\right) \leqq \delta_{V_{n}}\left(\frac{\Delta}{2}\right)
$$


and consequently

$$
\left|V_{n}\left(\bigcup_{j=n+1}^{\infty} E_{j}-E_{n}\right)\right| \leqq \frac{\Delta}{2} .
$$

It also follows from (3) that

$$
\lim _{n \rightarrow \infty} \mu\left(\bigcup_{j=n}^{\infty} E_{j}\right)=0 .
$$

Since $\left\{\bigcup_{l=j}^{\infty} E_{l}\right\}_{j=1,2, \ldots}$ is a decreasing sequence and $\mu\left(\bigcup_{l-2}^{\infty} E_{l}\right)$ $\leqq \frac{1}{2}<\infty$ by (3) we have from (5)

$$
\mu\left(\bigcap_{j=1}^{\infty} \bigcup_{l=j}^{\infty} E_{l}\right)=\lim _{l \rightarrow \infty} \mu\left(\bigcup_{l=j}^{\infty} E_{l}\right)=0
$$

Thus by the absolute continuity of the members of $C$ with respect to $\mu$ it follows that

$$
V_{n}\left(\bigcap_{j=1}^{\infty} \bigcup_{l=j}^{\infty} E_{l}\right)=0 \text { for all } n \geqq 1 .
$$

It now follows from (2), (4) and (6) that

$$
\begin{aligned}
\left|V_{n}\left(F_{n}\right)\right| & =\left|V_{n}\left(\bigcup_{j=n}^{\infty} E_{j}-\bigcap_{j=1}^{\infty} \bigcup_{l=j}^{\infty} E_{l}\right)\right| \\
& =\left|V_{n}\left(\bigcup_{j=n}^{\infty} E_{j}\right)-V_{n}\left(\bigcap_{j=1}^{\infty} \bigcup_{l=j}^{\infty} E_{l}\right)\right|=\left|V_{n}\left(\bigcup_{j=n}^{\infty} E_{j}\right)\right| \\
& =\left|V_{n}\left(E_{n} \cup\left(\bigcup_{j=n+1}^{\infty} E_{j}-E_{n}\right)\right)\right| \\
& =\left|V_{n}\left(E_{n}\right)+V_{n}\left(\bigcup_{j=n+1}^{\infty} E_{j}-E_{n}\right)\right| \\
& \geqq\left|V_{n}\left(E_{n}\right)\right|-\left|V_{n}\left(\bigcup_{j=n+1}^{\infty} E_{j}-E_{n}\right)\right| \\
& \geqq \Delta-\frac{1}{2} \Delta=\frac{1}{2} \Delta .
\end{aligned}
$$

But

$$
\begin{aligned}
\bigcap_{n=1}^{\infty} F_{n} & =\bigcap_{n=1}^{\infty}\left(\bigcup_{j=n}^{\infty} E_{j}-\bigcap_{j=1}^{\infty} \bigcup_{l=j}^{\infty} E_{l}\right) \\
& =\bigcap_{n=1}^{\infty}=\bigcup_{j=1}^{\infty} E_{j}-\bigcap_{j=1}^{\infty} \bigcup_{l=j}^{\infty} E_{l}=\phi
\end{aligned}
$$


and clearly $F_{n+1} \subset F_{n}$. Thus $\left\{F_{n}\right\}$ is a decreasing sequence of sets with empty intersection for which $V\left(F_{n}\right)$ does not converge to 0 uniformly in $V \in C$. This contradicts the assumption of equicontinuity from above at $\phi$ for $C$. Therefore the members of $C$ are uniformly absolutely continuous with respect to $\mu$ as we wished to prove. Q.E.D.

We note here that Theorem 1 holds for collections $C$ of finite complex valued signed measures. Indeed this follows immediately from Theorem 1 by considering the real and imaginary parts of such measures.

Proof of Theorem 2. Theorem 2 follows directly from Theorem 1 and the theorem quoted above from Halmos [2]. Q.E.D.

Now using the notation of Dunford and Schwartz [1] we have

Proof of Theorem 3. Without loss of generality $\mu$ may be assumed to be finite. To see this we note first that $\mu$ may be assumed to be $\sigma$-finite since only sequences of measurable functions are involved. Secondly, every $\sigma$-finite measure is equivalent to a finite measure. Now Theorem 3 follows from Theorem 1, the theorem quoted above from Dunford and Schwartz [1], and a theorem of Saks (Lemma IV, 9.7 of $[1])$.

\section{BIBLIOGRAPHY}

1. Nelson Dunford and Jacob T. Schwartz, Linear operators. I: General theory, Pure and Appl. Math., vol. 7, Interscience, New York, 1958. MR 22 \#8302. 504 .

2. Paul R. Halmos, Measure theory, Van Nostrand, Princeton, N. J., 1950. MR 11,

University of California, Berkeley, California 94720 